\title{
Deformation mechanism and microstructures on polycrystalline aluminum induced by high-current pulsed electron beam
}

\author{
CAI Jie ${ }^{1}$, JI Le $^{1}$, YANG ShengZhi ${ }^{1}$, WANG XiaoTong ${ }^{1}$, LI Yan $^{1}$, HOU XiuLi $^{1} \&$ \\ GUAN QingFeng ${ }^{1,2^{*}}$ \\ ${ }^{1}$ College of Materials Science and Engineering, Jiangsu University, Zhenjiang 212013, China; \\ ${ }^{2}$ Key Laboratory of Materials Modification by Laser, Ion and Electron Beams, Ministry of Education, Dalian University of Technology, \\ Dalian 116024, China
}

Received December 15, 2012; accepted February 5, 2013; published online May 3, 2013

\begin{abstract}
In this paper, we present an experimental investigation of deformation twinning in polycrystalline aluminum exposed to high-current pulsed electron beam (HCPEB) irradiation. The residual tensile stress with about $10^{2} \mathrm{MPa}$ was introduced in the irradiated surface layer. The feature characteristic irradiated with various numbers of pulses was investigated. The formation of a large number of twin bands on the surface irradiated with multiple pulses was determined. The experimental observations indicated that the deformation twinning was indeed triggered during HCPEB irradiation. It is suggested that high value of stress and strain rate induced by rapid heating and cooling due to HCPEB irradiation may cause the shifting of whole atomic planes simultaneously. Additionally, some slipping systems may be suppressed due to the geometric confinement by thinned size of surface layer, which can promote the initiation of deformation twinning.
\end{abstract}

high-current pulsed electron beam, pure aluminum, deformation, twinning, dislocation slipping

Citation: Cai J, Ji L, Yang S Z, et al. Deformation mechanism and microstructures on polycrystalline aluminum induced by high-current pulsed electron beam. Chin Sci Bull, 2013, 58: 2507-2511, doi: 10.1007/s11434-013-5848-5

High-current pulsed electron beams (HCPEB), a new type of surface modification techniques developed over the last decade [1-3], render the treated materials with nouveau properties such as high surface hardness and high wear and corrosion resistance [3]. Pogrebnjak and co-workers [4-8] described in detail the formation mechanisms of various dynamic effects induced by pulsed electron beam by investigating the modification of surfaces of single crystal stainless steel [4], titanium alloys [5], copper single crystal [6] and other metals and alloys [7,8]. They pointed out that during the transient bombardment process a high energy $\left(10^{6}-10^{9} \mathrm{~W} / \mathrm{cm}^{2}\right)$ is deposited only in a very thin layer (less than tens of micrometers) within a short time (a few microseconds) and causes superfast heating, melting, evaporation, and solidification. Such pulsed electron irradiation induces rapid heating and cooling in the surface and the formation

*Corresponding author (email: guanqf@ujs.edu.cn) of dynamic stress fields [1,2]. The combination of these processes makes it possible to form nouveau structural phenomena. Our previous studies have indicated that HCPEBs can serve as an effective platform for the investigation of the microstructures and mechanisms of high-speed deformation $[9,10]$. The characterization of deformation microstructures is very important for the development of our understanding of thermo-mechanical evolution during high-speed deformation.

Aluminum possesses higher stacking fault energy and aluminum alloys are widely applied structural materials. The surface modifications of polycrystalline aluminum by energetic particle beams have been investigated by many researchers [11,12]. Especially, our recent experimental evidence shown that even aluminum single crystal that is not normally associated with deformation twinning will twin by a proper HCPEB irradiation [9]. As we know, twinning is an important deformation mechanism in hexagonal 
closed-packed (hcp) metals. In body-centered-cubic (bcc) metals, it appears at low temperature where it becomes more favorable than dislocation based slip processes [13]. In faced-centered-cubic (fcc) metals, deformation twinning is more rare. It normally requires low temperatures and high stresses or strain rates. Of the fcc metals, pure aluminum was traditionally cited as a typical example of metals that do not exhibit deformation twinning at all because its stacking fault energy is too high [14].

In this paper, we present an experimental study of deformation twinning in polycrystalline aluminum under HCPEB irradiation using a Nadezhda-2 HCPEB device. A variety of pulse numbers were examined at about $4 \mathrm{~J} / \mathrm{cm}^{2}$ energy density. We observed that twinning deformation in the surface layer of pure aluminum was triggered after multiple-pulses HCPEB irradiation. This study focuses on the stress characteristic and the formation of deformation twins in the surface layer irradiated by HCPEB. The objective of this paper is to reveal the novel deformation behavior in polycrystalline aluminum induced by HCPEB irradiation which is very helpful for understanding the deformation mechanisms of metal at high-speed deformation.

\section{Experimental}

The pulsed electron beam irradiation was conducted using a Nadezhda-2 type HCPEB source. It produced an electron beam of low-energy $(10-40 \mathrm{keV})$, high peak current $\left(10^{2}-\right.$ $\left.10^{3} \mathrm{~A} / \mathrm{cm}^{2}\right)$, short pulse duration $(\sim 1.5 \mu \mathrm{s})$. The electron pulse was generated by an explosive emission graphite cathode. The accelerating voltage, magnetic field intensity and anode-collector distance controlled the beam energy density. More details on the principle of the HCPEB system were given in refs [1,2].

Bulk commercial pure aluminum was selected as the target material. Specimens were machined with sizes of 14 $\mathrm{mm}$ in length, $10 \mathrm{~mm}$ in width and $10 \mathrm{~mm}$ in height. One side surface of the aluminum specimen was mirror polished. The polished surfaces were irradiated at room temperature with 1,5 and 10 pulses using this HCPEB source, respectively. The HCPEB irradiation was carried out under the energy density of about $4 \mathrm{~J} / \mathrm{cm}^{2}$. Microstructural examinations were examined with an optical microscopy (OM) and a scanning electron microscopy (SEM). Grazing incidence $\mathrm{X}$-ray diffraction (GIXRD) measurements were carried out under filtered $\mathrm{Cu}$ k $\alpha$ irradiation by aid of a Rigaku D/max2400 diffractometer.

\section{Results}

Figure 1 shows the GIXRD curves which represent the diffraction peaks of $\mathrm{Al}$ (311) atomic plane obtained from the surfaces of aluminum samples irradiated by HCPEB with 1 ,

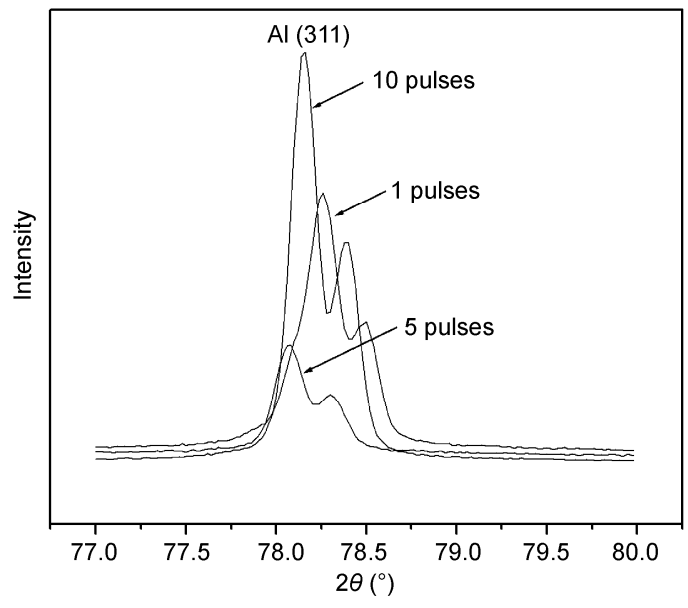

Figure 1 (311) diffraction peaks for aluminum samples irradiated by HCPEB with 1,5 and 10 pulses.

5 and 10 pulses, respectively. Based on [15], the values of deformation $(\varepsilon)$ and stress $(\sigma)$ could be estimated using the formulas:

$$
\varepsilon=\frac{d_{n}-d_{0}}{d_{0}}, \quad \sigma=\frac{1}{1-v} \varepsilon E,
$$

where $d_{n}$ is the $\mathrm{Al}$ lattice spacing (in plane of an irradiated sample), $d_{0}$ is the value of Al lattice spacing in an unstressed section, $v=0.330$ is Poisson's coefficient and $E=$ $7.0 \times 10^{4} \mathrm{MPa}$ is Young's modulus [15].

In unstressed sections, (311) lattice spacing values of $d_{0}$ $=0.12210 \mathrm{~nm}$ was obtained from PDF card of aluminum (65-2869). At the same time, the values of lattice spacing of $d_{n}=0.12216,0.12240$ and 0.12229 in (311) atomic plane of the irradiated samples with 1,5 and 10 pulses were determined from Figure 1, respectively. It is indicated that all the diffraction peaks of the aluminum samples irradiated by HCPEB shift towards low angle compare with the unstressed aluminum sample, suggesting that significant tensive macrostresses in irradiated surface layer were found in this study. The curve of residual stress values vs. pulsed number was plotted in Figure 2. It can be seen that the

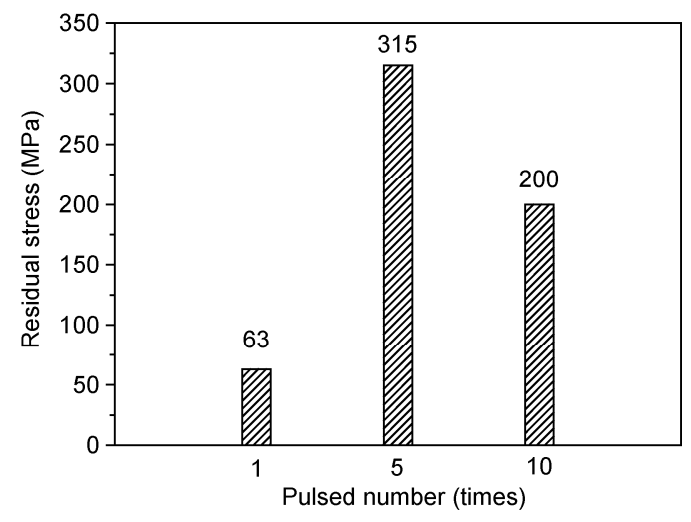

Figure 2 Residual stress distribution on irradiated surface. 
residual tress is $63 \mathrm{MPa}$ with 1 pulse, $315 \mathrm{MPa}$ with 5 pulses, and $200 \mathrm{MPa}$ with 10 pulses, suggesting that very high value of residual tensive stress is introduced within the surface layer after multiple pulses.

Figure 3 shows the surface morphology variations of polycrystalline aluminum irradiated by HCPEB. As indicated in Figure 3(a), there is no obvious deformation marking in the grain interior before HCPEB irradiation. After irradiation with 1 pulse, volcano-like craters appeared on the irradiated surface (marked by black arrows in Figure 3(b)). Craters were the most common feature on the surface, which is typical of many HCPEB irradiated metal surfaces and has been observed by many researchers [2,16]. From previous studies, such a morphology is the result of local sublayer melting and eruption through the solid outer surface [16]. In addition, it can be seen that severe deformations in the surface layer occurred after HCPEB irradiation. Dense slip bands were present in almost all the grains (marked by white arrows in Figure 3(b)). Especially, some slip bands intersected with each other, which resulted from the cross slip. This result reveals that dislocation slip is the main deformation mechanism after 1 pulse HCPEB irradiation.

After irradiation with 5 pluses, the deformation structures on the irradiated surface were completely changed. The craters became radial shape, as shown in Figure 3(c), displaying transverse expansion tendency. It appeared to be caused by the high value of tensile stress along surface radial direction during melting and solidification of the crater regions due to HCPEB irradiation. It is necessary to note that no slip bands were observed on the irradiated surface, whereas a mass of lens-like bands were formed in the interior of aluminum grains and these bands never cross the grain boundary. This crystallographic feature of lens-like bands is believed a typical morphology of deformation twins, suggesting deformation twinning turns into the dominate deformation mechanism on the irradiated surface of 5 pluses. Figure 3(d) shows the surface morphology after 10 pulses irradiation. One can see that the craters no longer exhibit radial shape due to relative low value of stress induced by 10 pulses comparing with its counterpart of 5 pulses. Moreover, the lens-like bands were evidently reduced. At same time, some dislocation slip lines parallel with the lens-like bands also emerged, indicating that the shear planes of lens-like bands and dislocation slip lines are both the close-packed plane of fcc structure, i.e. (111) plane in nature. It is completely in conformity with the crystallographic features of deformation twinning in fcc metals. Figure 3(d) illustrates that a mixed structure with both lens-like bands and dislocation slip lines was obtained simultaneously on the irradiated surface with 10 pulses, corresponding to the mixed deformation mechanisms of both deformation twinning and dislocation slipping.

Figure 4(a) and (b) show the SEM images of lens-like structure on the surface of polycrystalline aluminum irradiated by HCPEB with 5 pulses and 10 pulses, respectively. The magnification image of the lens-like reveals that the bands are regularly parallel with a median ridge in it, which is a typical feature caused by shear deformation. Figure 4 provides further evidence for the fact that the lens-like bands are deformation twins induced by intense shear deformation in nature. The results mentioned above demonstrate
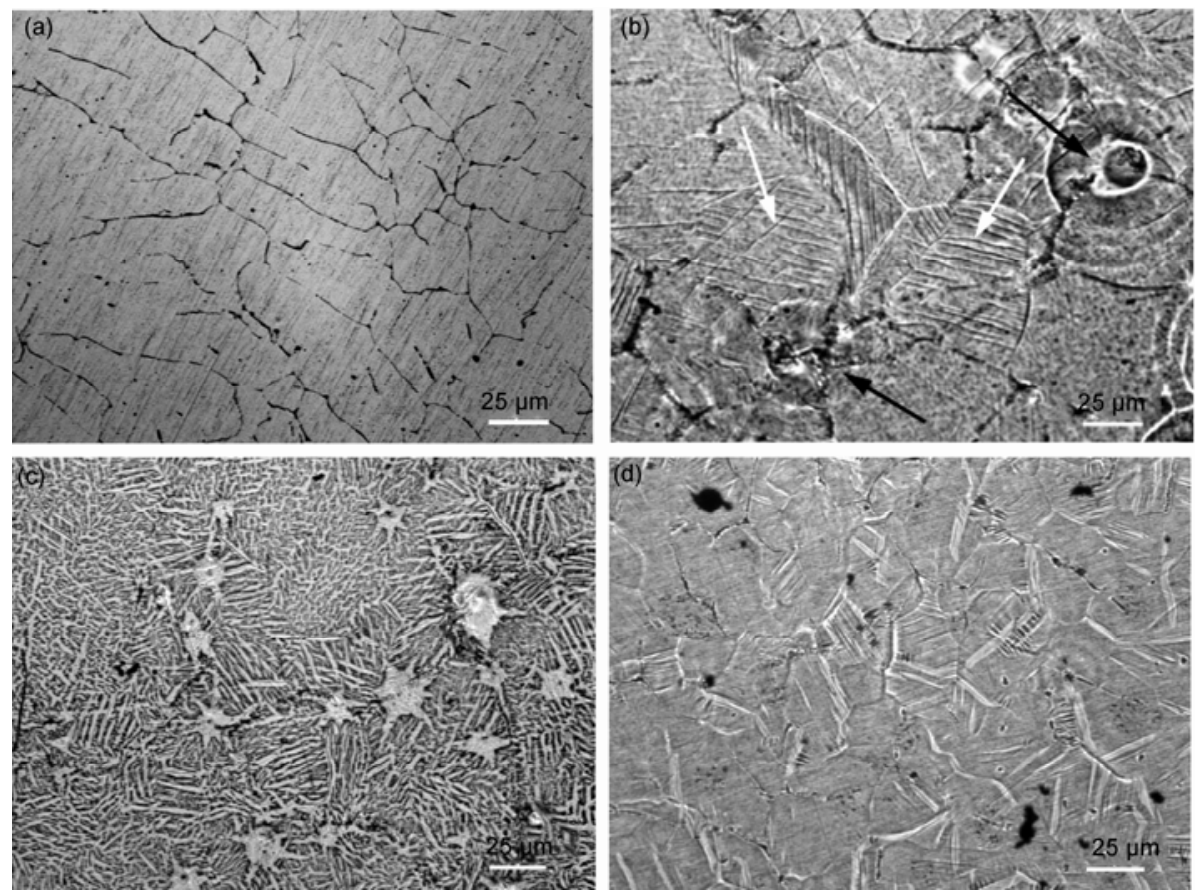

Figure 3 Surface morphology of polycrystal pure aluminum irradiated by HCPEB. (a) Pre irradiation, (b) 1 pulse, (c) 5 pulses, (d) 10 pulses. 

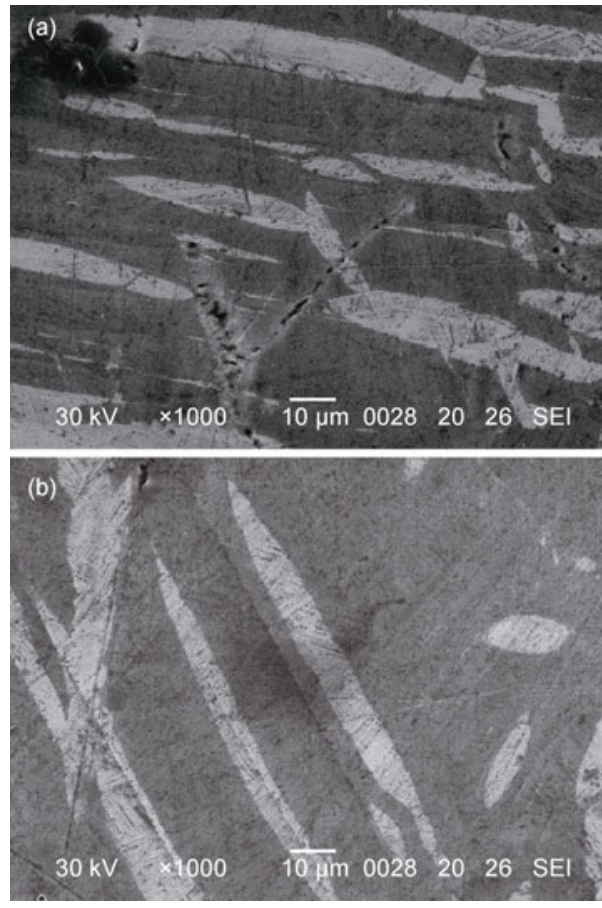

Figure 4 SEM images of the twins on sample surface after multi HCPEB irradiation. (a) 5 pulses, (b) 10 pulses.

that HCPEB irradiation can induce deformation twinning on the surface layer of pure aluminum.

\section{Discussion}

Dislocation slipping and twinning are the two primary deformation mechanisms. Moreover, they are competing processes [14]. Consequently, once dislocation slipping is triggered successfully in the crystal, twinning is inhibited correspondingly. Only when applied stress cannot effectively activate dislocation slipping, the deformation twinning will initiate. However, twinning mechanisms in fcc metals involve glide of Shockley partial dislocations (twinning dislocations with Burgers vector $b_{\text {twin }}=1 / 6 a_{0}\langle 112\rangle$, where $a_{0}$ is the fcc lattice constant) on successive $\{111\}$ planes to create multi-layered faults that could then produce a twin [17]. Therefore, twinning initiates in fcc metals depending on the intrinsic stacking fault energy. Based on conventional deformation mechanisms, pure aluminum does not exhibit deformation twinning at all because its stacking fault energy is too high.

The deformation twinning has been discovered in high purity polycrystalline aluminum by experimental observation [18]. The twins were accidentally observed in the frontier of edge type fracture of a thin film TEM sample, and grew along the $\langle 112\rangle$ direction of $\{111\}$ twinning plane, which were attributed to the release of $1 / 6\langle 121\rangle$ partial dislocation on $\{111\}$ twinning plane before fracture. Recently, in the condition of geometric size being restricted, such as nanocrystalline aluminum, deformation twinning was also discovered $[19,20]$. They also ascribed the twins to the release of partial dislocations in nanocrystalline aluminum grain boundary. Our previous experimental results also indicated that aluminum single crystal should twin by a proper HCPEB irradiation [9].

Recently, Kiritani et al. [21] reported that a large number of vacancies were produced during high-speed heavy plastic deformation of thin foils of fcc metals. They observed both twins and a large density of stacking fault tetrahedra (SFTs) but very low dislocation densities in aluminum foil after deformation, whereas this result did not present in bulk aluminum. As a possible explanation, they propose a dislocation-free deformation mechanism, although this theory has not gained wide acceptance [22]. According to conventional dislocation slipping mechanism, when applied stress is imposed to the atoms in the vicinity of dislocation core, even a small stress level is enough to cause the movement of these atoms to nearby stable positions. It is because the atoms are in a high stress state already. In plastic deformation dominated by dislocation slipping, internal stress can relax automatically by dislocation slipping, which reduces the accumulated internal stress in the plastic deformed material and leads to higher strain. Whereas, according to the dislocation-free deformation mechanism proposed by Kiritani et al. [21,22], deformation is not carried out by dislocation slipping. Thus, the elastic deformation must begin to increase correspondingly, accompanied by the increase of internal stress. When the internal stress increases to be high enough, all of the atoms will be in an unstable state, and tend to move towards next stable position by overcoming the potential barrier constituted by the neighboring atoms. In this situation, the moving atoms are not limited to the individual atoms in the vicinity of dislocation core, but all of them in the whole atomic plane are involved. That is the atoms move in the way of atomic plane. For the slip mechanism, the displacement of atoms is accomplished by atomic lines (dislocations). It is the fundamental difference between two mechanisms. If the atoms on $\{111\}$ close-packed plane move along or near the $\langle 111\rangle$ direction simultaneously, twinning can occur once the atomic plane is misarranged. This mechanism could well explain our experimental results.

Besides experimental observation, the recent computer simulation studies of molecular dynamics indicated that while the grain size of pure aluminum reduces to nanometer scale, twinning can be induced at a certain applied stress level. However, for the bulk pure aluminum without any structural limitation twinning cannot occur [23]. Jin et al. [24] investigated the nano-indentation on (001) plane of two-dimensional single crystal aluminum by multiscale quasi continuous model. The results reveal that in spite of the symmetry of aluminum is high, and the geometry size restricts the activation of some sliding systems, which is likely to lead to the suppression of dislocation slipping. This 
effect promotes the occurrence of deformation twinning. It is similar to the low symmetry structure, for example hcp crystal, that prone to deformation twinning due to the initiations of dislocation slipping fail to be satisfied easily. In the present experimental conditions, the high tensile stress on the irradiated surface is only confined to the surface layer within a few microns [9-11], which is likely to impede the initiation of some slipping systems and cause the suppression of dislocation slipping. The twins structure observed in irradiated surface layer of pure aluminum in this paper may be an implication.

\section{Conclusions}

In this study, HCPEB irradiation was adopted to investigate the high speed deformation behavior of polycrystalline aluminum, which provides a means to access atomic interactions in materials and probe the details of bonding and defect physics. The XRD results show that the residual tensile stress with about $10^{2} \mathrm{MPa}$ has been introduced in the irradiated surface layer. The stresses introduced by HCPEB irradiation play an important role for the deformation mechanism of irradiated surface. The observations of surface deformation structure reveal that deformation mechanism of the surface layer with 1 pulse irradiation is dominated by dislocation slipping. After 5 pulses, the deformation mechanism turns into deformation twinning. For 10-pulses, the deformation structures on irradiated surface consisted of both twin bands and dislocation slip lines, suggesting that the deformation mechanism in this situation is governed by both twinning and dislocation slipping. It is suggested that the stress with high value and strain rate induced by rapid heating and cooling due to HCPEB irradiation could cause the shifting of whole atomic planes simultaneously. Additionally, some slipping system may be suppressed due to the geometric confinement by thinned size of surface layer, which can promote the initiation of deformation twinning.

This work was supported by the National Natural Science Foundation of China (U1233111, 50671042) and Open Foundation of Key Laboratory of Materials Modification by Laser, Ion and Electron Beams, Ministry of Education, Dalian University of Technology (DP1051102).

1 Proskurovsky D I, Rotshtein V P, Ozur G E, et al. Pulsed electronbeam technology for surface modification of metallic materials. J Vac Sci Technol A, 1998, 16: 2480-2488

2 Proskurovsky D I, Rothtein V P, Ozur G E, et al. Physical foundations for surface treatment of materials with low energy, high current electron beams. Surf Coat Technol, 2000, 125: 49-56

3 Zou J X, Zhang K M, Hao S Z, et al. Mechanisms of hardening, wear and corrosion improvement of $316 \mathrm{~L}$ stainless steel by low energy high current pulsed electron beam surface treatment. Thin Solid
Films, 2010, 519: 1404-1415

4 Pogrebnjak A D, Lebed A G, Ivanov Y F. Modification of single crystal stainless steel structure (Fe-Cr-Ni-Mn) by high power ion beam. Vacuum, 2001, 63: 483-486

5 Pogrebnjak A D, Kobzev A P, Gritsenko B P, et al. Effect of Fe and $\mathrm{Zr}$ ion implantation and high-current electron irradiation treatment on chemical and mechanical properties of Ti-V-Al alloys. J Appl Phys, 2000, 87: 2142-2148

6 Tyurin Y N, Pogrebnjak A D. Advanced in the development of the technologies and equipment for coating deposition. Surf Coat Technol, 1999, 111: 269-275

7 Pogrebnjak A D, Bratushka S, Boyko V I, et al. A review of mixing processes in $\mathrm{Ta} / \mathrm{Fe}$ and $\mathrm{Mo} / \mathrm{Fe}$ systems treated by high current electron beams. Nucl Instrum Methods Phys Res Sect B-Beam Interact Mater Atoms, 1998, 145: 373-390

8 Pogrebnjak A D, Ladysev V S, Pogrebnjak N A, et al. A comparison of radiation damage and mechanical and tribological properties of $\alpha-\mathrm{Fe}$ exposed to intense pulsed electron and ion beams. Vacuum, 2000, 58: 45-52

9 Guan Q F, Zhang Q Y, Dong C, et al. Deformation twining in singlecrystal aluminum induced by high-current pulsed electron beam. $\mathrm{J}$ Mater Sci, 2005, 40: 5049-5052

10 Peng D J, Guan Q F, Chen B, et al. Deformation twinning in pure nickel induced by a high-current pulsed electron beam. Arab J Sci Eng, 2011, 36: 663-669

11 Guan Q F, Cheng D Q, Qiu D H, et al. The vacancy defect clusters in polycrystalline pure aluminum induced by high-current pulsed electron beam. Acta Phys Sin, 2009, 58: 4846-4852

12 Pogrebnjak A D, Ruzimov S M. Increased microhardness and positron annihilation in $\mathrm{Al}$ exposed to a high-power ion beam. J Phys Lett A, 1987, 120: 259-261

13 Mao W M, Chen L, Yu Y N. Influence of reaction stresses induced by dislocation slips on the orientation evolution in bcc metals. Chin Sci Bull, 2003, 48: 204-207

14 Venables J. Deformation twinning in fcc metals. In: Reed-Hill R E, ed. Proceedings of the Metallurgical Society Conference. New York: Gordon and Breach Science Publishers, 1963. 77-78

15 Pinegyn V I, Zubarev E N, Kondratenko V V, et al. Structure and stressed state of molybdenum layers in Mo/Si multilayers. Thin Solid Films, 2008, 516: 2973-2980

16 Qin Y, Wang X G, Dong C, et al. Temperature field and formation of crater on the surface induced by high current pulsed electron beam bombardment. Acta Phys Sin, 2003, 52: 3043-3048

17 Kibey S, Liu J B, Johnson D D, et al. Predicting twinning stress in fcc metals: Linking twin-energy pathways to twin nucleation. Acta Mater, 2007, 55: 6843-6851

18 Christian J W, Mahajan S. Deformation twinning. Prog Mater Sci, 1995, 39: 1-157

19 Chen M W, Ma E, Hemker K J, et al. Deformation twinning in nanocrystalline aluminum. Science, 2003, 300: 1275-1277

20 Liao X Z, Zhou F, Lavernia E J, et al. Deformation twins in nanocrystalline Al. Appl Phys Lett, 2003, 83: 5062-5064

21 Kiritani M, Sota T, Tawara T, et al. Defect structures introduced in fcc metals by high-speed deformation. Radiat Eff Defects Solids, 2002, 157: 53-74

22 Kiritani M, Satoh Y, Kizuka Y, et al. Anomalous production of vacancy clusters and the possibility of plastic deformation of crystalline metals without dislocations. Philos Mag Lett, 1999, 79: 797-804

23 Tsuru T, Shibutani Y. Anisotropic effects in elastic and incipient plastic deformation under (001), (110), and (111) nanoindentation of $\mathrm{Al}$ and $\mathrm{Cu}$. Phys Rev B, 2007, 75: 035415

24 Jin J, Shevlin S A, Guo Z X. Multiscale simulation of onset plasticity during nanoindentation of Al (001) surface. Acta Mater, 2008, 56: 4358-4368

Open Access This article is distributed under the terms of the Creative Commons Attribution License which permits any use, distribution, and reproduction in any medium, provided the original author(s) and source are credited. 